Z Gerontol Geriat 2012 · 45:584-584

DOI 10.1007/s00391-012-0398-z

Online publiziert: 1. September 2012

๑) Springer-Verlag 2012

\author{
C. Kricheldorff ${ }^{1} \cdot$ F. Oswald ${ }^{2}$ \\ 1 Soziale Arbeit in gerontologischen Arbeitsfeldern und im Gesundheitswesen, \\ Katholische Hochschule Freiburg \\ 2 Interdisziplinäre Alternswissenschaft, Fachbereich Erziehungswissenschaften, \\ Goethe-Universität Frankfurt am Main
}

\title{
Wert(e) des Alters
}

Im vorliegenden Heft greifen wir die Welche Wertvorstellungen über das Alter bestehen und welche Werte sich mit dem Alternsprozess verbinden, ist einerseits Ausdruck biographischer Prägungen und abhängig von persönlichen Werthaltungen. Andererseits bestimmen auch die jeweiligen gesellschaftlichen Realitäten maßgeblich mit, welcher Wert dem Alter als Lebensphase und dem alten Menschen an sich zugestanden wird. Werte prägen gesellschaftliche Altersbilder und die damit verbundenen Rollenerwartungen an die Alten sowie Erwartungen an das eigene Altern. Der erlebte Selbstwert älterer und alter Menschen ist eng verknüpft mit der Wertschätzung, die sie aus ihrer Umwelt erfahren. Und nicht zuletzt ist in einer Gesellschaft des langen Lebens das wirtschaftliche Potenzial des Alters auch eine Ressource für gesellschaftliche Wertschöpfung. Das Thema „Wert(e) des Alters" kennt also viele Facetten und Ausprägungen.

Nicht zuletzt die damit verbundene Themenvielfalt war Anlass für die gemeinsame Jahrestagung der Sektionen III und IV der DGGG, die vom 22. bis 23. September 2011 in Frankfurt am Main zu diesem Tagungsmotto stattfand. Die außerordentlich positive Resonanz, die sich in der großen Zahl der eingereichten Beiträge und Besucher widerspiegelte, macht die Bedeutung des Themas für die Gerontologie und darüber hinaus offenkundig. Die Tagungsteilnehmer beschäftigten sich in 30 Postern und über 80 mündliche Beiträgen einerseits mit ökonomischen und ethischen Aspekten des Alters und Alterns, andererseits aber auch mit Themen wie Technik, Arbeit, Lebensqualität, Wohnen und Pflege. Vorträge der Keynote-Speaker und einige weitere Vorträge noch einmal auf und bilden mit den mittlerweile daraus entstandenen Beiträgen einen Themenschwerpunkt, der wichtigen Aspekten aus der Tagung Raum gibt, auch wenn längst nicht die ganze thematische Vielfalt und Fülle angemessen abgebildet werden kann.

Im Editorial fragt $K$. Aner danach, was uns die Alten heute wirklich wert sind und wie sie sich selbst sehen. Den Anfang der Einzelbeiträge machen $F$. Kolland und R.A.Meyer-Schweizer, die in ihrem Beitrag "Altern und Wertewandel“" aufzeigen, wie sich normative Vorgaben der Gesellschaft wandeln, hin zu mehr Freiheit und Autonomie und welche Folgen und Widersprüche sich daraus für das Altern und insbesondere auch für die Alternsforschung heute ergeben können. Der Beitrag von F. Schulz-Nieswandt beschäftigt sich mit der Ausgrenzung des Fremdartigen, des Andersseins, wie Krankheit, Alter und Behinderung, als Ausdruck einer Art kulturellen Grammatik. „Thesen zur kritischen Gerontologie aus sozialwissenschaftlicher Sicht" stellt D. Köster auf und legt damit das Fundament für den neu gegründeten Arbeitskreis Kritische Gerontologie, der sich im Rahmen der Frankfurter Tagung konstituiert hat. Die „Wirtschaftskraft Alter“ steht im Mittelpunkt des Beitrags von U. Fachinger, der sich mit monetären Werten, also den Aspekten Vermögen, Einkommen und Ausgaben, älterer und alter Menschen auseinandersetzt. Mit den Folgen der „Ökonomisierung von Pflege in Deutschland, Schweden und Großbritannien " beschäftigt sich D. Auth. Ebenfalls mit dem Thema Pfle- ge, aber aus der Sicht von drei Fallbeispielen zu individuellen und sozialräumlichen Ressourcen älterer Menschen, tragen unter dem Titel „Selbstbestimmung bei Pflegebedürftigkeit" K. Kammerer et al. bei. Den Abschluss machen S. Schäper und S. Graumann mit einem Beitrag, der beschreibt, wie ältere Menschen mit geistiger Behinderung das „Alter(n) als wertvolle Lebensphase erleben" können und welche Herausforderungen und Bedingungen damit verbunden sind.

Allen Beitragenden und nicht zuletzt auch allen Teilnehmenden der Frankfurter Tagung sei an dieser Stelle herzlich gedankt. Uns ist bewusst, dass wir mit der vorliegenden Auswahl nur einen Ausschnitt zeigen können, der einen mehr oder weniger breiten thematischen Querschnitt darstellt. Aber wenn es uns gelingt, damit eine durchaus kontrovers geführte Debatte zum Thema „Wert(e) des Alters“ weiter voranzubringen, hat dieser Themenschwerpunkt sein Hauptziel erreicht.

\section{Korrespondenzadressen}

Prof. Dr. C. Kricheldorff

Soziale Arbeit in gerontologischen

Arbeitsfeldern und im Gesundheitswesen, Katholische Hochschule Freiburg Karlstr. 63, 79104 Freiburg i. Br. cornelia.kricheldorff@kh-freiburg.de

\section{Prof. Dr. Frank Oswald}

Interdisziplinäre Alternswissenschaft Fachbereich Erziehungswissenschaften Goethe-Universität Frankfurt am Main Robert-Mayer-Str. 1, 60325 Frankfurt am Main oswald@em.uni-frankfurt.de 